\title{
SÍNDROME HEREDITÁRIA FAMILIAR ASSOCIADA A NEOPLASIAS: AÇÃO EXTENSIONISTA NA CONSTRUÇÃO DE HEREDOGRAMAS E DETECÇÃO DE COMORBIDADES
}

A. S. Gondim ${ }^{1}$, C. E. L. Soares ${ }^{2}$, M. P. Custódio ${ }^{3}$, A. R. de Castro ${ }^{4}$; F. E. L. Silva ${ }^{5}$ \& A. R. P. Quidute ${ }^{6}$

1Bolsista do Núcleo de Estudos e Pesquisa em Tumores Neuroendócrinos Esporádicos e Associados a Síndromes Familiares (NEPTUNEF) e graduanda em Medicina pela Universidade Federal do Ceará (UFC). E-mail: andressagondim@ outlook.com; 2 Bolsista do Núcleo de Pesquisa e Desenvolvimento de Medicamentos (NPDM), membro do NEPTUNEF e graduando em Medicina pela UFC. E-mail: eduardolopes.ti@gmail.com; 3 Bolsista em Iniciação Científica pela CNPq, membro do NEPTUNEF, graduanda em Medicina pela UFC. E-mail: marinapcustodio5@gmail.com; 4 Membro do NEPTUNEF e graduanda em Medicina pela UFC. E-mail:

alexialelecastro@gmail.com; 5Bolsista em Iniciação Acadêmica, membro do NEPTUNEF e graduando em Medicina pela Universidade

Federal do Ceará (UFC). E-mail: emanuellima16.16@gmail.com; 6 Coordenadora da ação extensionista do Núcleo de Estudos em

Tumores Neuroendócrinos Esporádicos e Associados a Síndromes Familiares (NEPTUNEF), pesquisadora afiliada ao Laboratório de

Farmacogenética (FARMAGEN) do Núcleo de Pesquisa e Desenvolvimento de Medicamentos (NPDM) e professora do Departamento de Fisiologia e Farmacologia (DFF) da UFC. E-mail: arquidute@gmail.com

Artigo submetido em setembro/2018

\section{RESUMO}

A Neoplasia Endócrina Múltipla Tipo 1 (NEM-1) é doença hereditária $(\mathrm{DH})$ caracterizada pela presença de hiperparatireoidismo (HPT), adenoma de hipófise (AH) e tumor gastroenteropancreático (TGEP). A análise de heredogramas (HDGs) é estratégico nas DH. O presente trabalho objetiva construir, analisar HDGs e detectar comorbidades na NEM-1. Para tanto, utilizamos entrevista, aplicação de questionário, criação de HDGs (GenoPro®) e avaliação de parâmetros metabólico em parceria com o NPDM/HUWC-UFC. Foram analisados 33 casos distribuídos em 3 famílias (F). Na F1, 18 casos:11 HPT, 10 AH e 08 TGEP. F2, 5 casos: 3 HPT, 3 TGEP e 1 AH. F3, 10 casos: 3 HPT, 8 AH e 4 TGEP. Detectamos um antecessor comum entre 2 famílias e presença de surdo-mudez congênita. Em 17 indivíduos, 10 mulheres, de diferentes famílias, observamos $41 \%$ de humor deprimido, $35 \%$ obesos (OB) e 59\% de sobrepeso (SP). Concluímos que os HDGs permitiram caracterizar o perfil intrafamiliar, relacionar famílias aparentemente distintas, com frequência elevada de alteração do humor, OB e SP.

PALAVRAS-CHAVE: Heredograma. Tumores neuroendócrinos. NEM-1. Sobrepeso.

\section{HEREDITARY FAMILY SYNDROME ASSOCIATED WITH NEOPLASMS: EXTENSIONIST ACTION CONSTRUCTING HEREDOGRAMS AND DETECTING COMORBITIES}

\begin{abstract}
The Multiple Endocrine Neoplasia Type 1 (MEN-1) is a hereditary disease (HD) characterized by hyperparathyroidism (HPT), pituitary adenoma (PA) and gastroenteropancreatic tumor (GEPT) presence. The analysing heredograms (HDGs) is strategic in HD. The aims of this manuscrip are to construct, analyze HDGs and detect comorbidities in MEN-1. Methodology: Interview, application of questionnaire, creation of HDGs (GenoProß) and evaluation of metabolic parameters devevloped at NPDM/HUWCUFC. There were analyzed 33 cases distributed in 3
\end{abstract}

families (F). In F1, 18 cases: $11 \mathrm{HPT}, 10 \mathrm{PA}$ and 08 GEPT. F2, 5 cases: 3 HPT, 3 GEPT and 1PA. F3, 10 cases: 3 HPT, 8 PA and 4 GEPT. There was detected that common predecessor between 2 families and congenital deaf-mute presence. In 17 individuals, 10 were women from different families, observed $41 \%$ of depressed mood, 35\% obese (OB) and 59\% overweight $(\mathrm{OW})$. HDGs allowed us to characterize the intrafamilial profile, to relate apparently different families. There was a high frequency of mood alterations, $\mathrm{OB}$ and $\mathrm{OW}$. 


\section{INTRODUÇÃO}

As doenças genéticas são enfermidades causadas por alterações no material genético dos indivíduos, seja por uma mutação, deleção ou outro defeito. São condições de preocupação no serviço médico em razão da possibilidade de afetar vários membros da mesma família, ser herdada nas futuras gerações e ainda poderem ser manifestadas diferentemente em cada indivíduo. Essas doenças merecem, portanto, uma observação mais rigorosa do Sistema Único de Saúde (SUS) na capacitação das equipes de saúde para o diagnóstico e seguimento desses pacientes.

O Serviço de Endocrinologia e Diabetes (SED) do Hospital Universitário Walter Cantídio (HUWC), dentre todos as suas atividades, conta com o atendimento de pacientes com doenças endócrinas genéticas, como a Neoplasia Endócrina Múltipla tipo 1 (NEM-1), sendo, de fundamental importância, a utilização de mecanismos para o estudo dos familiares de portadores da patologia, facilitando o diagnóstico precoce e favorecendo um melhor prognóstico. Enquanto isso, o projeto de extensão Núcleo de Estudos e Pesquisa em Tumores Neuroendócrinos Familiares e Associados a Síndromes Familiares (NEPTUNEF), se volta ao atendimento multidisciplinar integrado dos pacientes portadores de Tumores Neuroendócrinos esporádicos e familiares e tem como objetivos específicos: integrar assistência, ensino e pesquisa entre as diferentes especialidades responsáveis pelo seguimento clínico e terapêutico dos pacientes; contribuir para a formação de internos, residentes, graduandos e pós-graduandos; promover um diagnóstico mais precoce; favorecer a redução da morbimortalidade e dos custos com a saúde no âmbito do SUS; e desenvolver linhas de pesquisa, contribuindo assim na formação de novos grupos de pesquisa e desenvolvimento profissional na nossa instituição, o HUWC.

A Neoplasia Endócrina Múltipla Tipo 1 (NEM-1) é uma doença hereditária de padrão autossômico dominante. Caracteriza-se pela presença de uma ou mais das seguintes comorbidades: hiperparatireoidismo (HPT), adenoma de hipófise (AH) e tumores gastroenteropancreático (TGEP). A NEM-1 é causada por uma mutação que inativa o gene MEN1, que codifica a proteína menin, um supressor tumoral, em que $80 \%$ dos pacientes apresentam manifestação clínica entre a terceira e a quinta década de vida. Essa doença tem chamado atenção no ambulatório de Endocrinologia do HUWC em razão da grande quantidade de pacientes que vem sendo diagnosticados através desse serviço. 
O SED que, atualmente, acompanha aproximadamente 98 pacientes diagnosticados ou em investigação de NEM-1, com o auxílio do NEPTUNEF, tem conseguido grandes evoluções para um diagnóstico mais precoce dessa condição por meio do screening familiar e da criação de heredogramas, que permitem a melhor observação e entendimento de como a doença tem se manifestado nos pacientes, quais comorbidades são mais prevalentes e uma melhor organização da distribuição de casos dentro das famílias.

O diagnóstico precoce, normalmente, possibilita bons resultados tanto no tratamento clínico quanto cirúrgico. A criação de heredogramas é, então, necessária para a criação de um mapa geral de cada família indicando os pacientes já afetados e indicando aqueles que podem manifestar a doença futuramente, atribuindo o screening familiar.

A obesidade tem alcançado nas últimas décadas um índice alarmante, afetando tanto países desenvolvidos como em desenvolvimento e sendo considerada uma epidemia. Essa adiposidade exacerbada é relacionada ao aumento da prevalência de hipertensão arterial, sedentarismo, diabetes mellitus, hipercolesterolemia, doenças cardiovasculares e cânceres, afirmam CUNHA et al. (2010), e hoje já é tida como fator predisponente aos cânceres de mama, cólon, reto, pâncreas, fígado e vesícula biliar, entre outros, sendo a manutenção do peso ideal meio de reduzir o risco de uma gama de cânceres. Sabendo disso, é importante lembrar o risco geneticamente aumentado de tumores gastroenteropancreáticos em portadores da NEM-1 e visar, desse modo, a importância do controle de outros fatores de risco para o desenvolvimento dessas neoplasias.

No âmbito da detecção de comorbidades, o Índice de Massa Corporal (IMC) e a circunferência abdominal entram como ferramentas de baixíssimo custo e fácil aferição, podendo facilmente ser adicionadas na rotina de acompanhamento médico não só de indivíduos portadores de NEM-1, mas de todos os pacientes em geral.

\section{BASES TEÓRICAS DA IMPORTÂNCIA DA CONSTRUÇÃ̃o DE HEREDOGRAMAS E DA DETECÇÃO DE SOBREPESO}

A utilização de heredogramas vem sendo uma importante ferramenta no melhor gerenciamento e acompanhamento de famílias acometidas por doença hereditária,

principalmente envolvendo câncer. Sendo citado por LAWALL (2012, p. 459) como uma ferramenta que facilita a identificação de padrões de herança e comportamento da doença que 
“por ser universal, qualquer pessoa que possua conhecimento acerca da simbologia que compõe o heredograma é capaz de interpretá-lo, o que o torna acessível e passível de utilização em pesquisas estatísticas. Para construí-lo, a história familiar deve ser obtida por meio de questionários ou entrevistas que incluem não só os membros afetados (com câncer), mas também os membros não afetados, que, para o olhar médico, são os indivíduos em risco de desenvolver a doença."

Por essa razão, a criação de heredogramas deve ser um dos conhecimentos médicos e ser aplicado ao seu cotidiano clínico em meio a abordagem de pacientes com síndromes genéticas, pois, dependendo do padrão de herança da doença, outros familiares poderão também ser diagnosticados graças a um estudo familiar mais aprofundado.

Ferramentas de baixo custo como o software GenoPro® garantem uma maior facilidade na confecção desses heredogramas e ainda possibilitam uma melhor identificação de cada descrição das síndromes. Utilidades como essa devem ser implementadas nos serviços de atendimento do SUS como forma de aperfeiçoamento clínico em consultas, pois, além da praticidade, podem favorecer o diagnóstico mais cedo em pacientes portadores silenciosos dos mais diversos tipos de patologias hereditárias.

A limitação ocorre quando se verifica a falta de capacitação e conhecimento dos profissionais em saúde dessas ferramentas, assim como outras mais que poderiam auxiliar em suas consultas devido à maior facilidade de compreensão de doenças genéticas e acompanhamento de famílias portadoras. Por isso, é necessária uma maior implementação de ferramentas digitais no ambiente hospitalar e ambulatorial dos hospitais do SUS, além de uma maior capacitação de médicos e demais profissionais para o uso desses recursos em prol da manutenção e atualização do serviço clínico com relação às síndromes genéticas.

No que concerne à obesidade, é conhecido seus impactos sobre a vida do ser humano, a inclinação promovida para demais comorbidades e seu abalo sobre os gastos de saúde pública e privada. Tendo em vista tal panorama, a detecção precoce do sobrepeso se torna fundamental.

A Organização Mundial de Saúde define como baixo peso o indivíduo com o IMC < $18,5 \mathrm{~kg} / \mathrm{m}^{2}$, peso ideal o com o IMC $\geq 18,5 \mathrm{~kg} / \mathrm{m}^{2}$ e $<25 \mathrm{~kg} / \mathrm{m}^{2}$, sobrepeso a pessoa com o IMC $\geq 25 \mathrm{~kg} / \mathrm{m}^{2}$ e $\leq 29,9 \mathrm{~kg} / \mathrm{m}^{2}$, e a obesidade como o IMC $\geq 30 \mathrm{~kg} / \mathrm{m}^{2}{ }^{1,2}$

\footnotetext{
${ }^{1}$ World Health Organization. Body mass index classification - report of a WHO consultation on obesity. Geneva: WHO, 1995. Technical Report Series 854.

${ }^{2}$ World Health Organization. Obesity: preventing and managing the global epidemic. Report of a World Health Organization Consultation. Geneva: World Health Organization, 2000. p. 256. WHO. Technical Report Series 284.
} 
Cunha et al. (2010) compara o rendimento laboral de trabalhadores obesos e não obesos, tendo, esses primeiros, menor rendimento no trabalho, devido à menor desenvoltura nesse contexto, maior perda de dias por déficits de saúde e restrições mais consideráveis em comparação aos trabalhadores não obesos. Como consequência, advêm desse fato prejuízos tanto financeiros quanto emocionais para o indivíduo, além de gastos em saúde que poderiam ser evitados.

A hiperinsulinemia, a elevada biodisponibilidade de hormônios esteroides e as inflamações advindas da obesidade, provocam por meio de diversos processos o aumento do risco de cânceres, como o de cólon, e, provavelmente, outros tumores, tais como os de pâncreas e de fígado, afirmam Calle e Kaaks (2004) com base em evidências de uma gama de estudos epidemiológicos e experimentais. Essa hiperinsulinemia provém da resistência à insulina devido aos altos níveis séricos de ácidos graxos livres, liberados, sobretudo, da gordura visceral, continuam os autores.

De acordo com Peixoto et al. (2006),

Apesar de não medir a composição corporal, o IMC possui bom potencial como indicador do estado nutricional em estudos epidemiológicos. Sua utilização se baseia nos resultados de estudos populacionais que mostram que o IMC é pouco correlacionado com a altura e altamente correlacionado com a massa de gordura absoluta e na associação existente entre IMC elevado e morbidade e mortalidade por doenças cardiovasculares, diabete melito, câncer de cólon e doenças das vias biliares.

Tabela 1 - Relação IMC e comorbidades

\begin{tabular}{ccc}
\hline IMC $\left(\mathbf{k g} / \mathbf{m}^{\mathbf{2}}\right)$ & Classificação & Risco de comorbidades \\
\hline $\mathbf{1 1 8 , 5}$ & Baixo peso & Baixo \\
\hline $\mathbf{1 8 , 5}$ a 24,9 & Peso normal & Médio \\
\hline $\mathbf{2 5}$ a 29,9 & Pré-obeso & Aumentado \\
\hline $\mathbf{3 0}$ a 34,9 & Obesidade grau I & Moderado \\
$\mathbf{3 5}$ a 39,9 & Obesidade grau II & Grave \\
$\mathbf{2 4 0}$ & Obesidade grau III & Muito grave \\
& Fonte: & Organização Mundial de Saúde (2000).
\end{tabular}

Já a circunferência abdominal (CA), também chamada de circunferência de cintura (CC), mede a circunferência do ponto médio entre a crista ilíaca e o rebordo costal inferior, região em que pode se concentrar a gordura visceral. A Organização Mundial de Saúde (OMS) tem como valores ideias para homens a CA inferior a $94 \mathrm{~cm}$ e, para mulheres, inferior a $80 \mathrm{~cm}$.

Adiciona-se a isso, a afirmação de Anjos et al. (1992) de que "O IMC também se 
correlaciona em níveis altos com a circunferência abdominal ( $\mathrm{r}$ em torno de 0,80 ) como demonstrado no estudo longitudinal de Framingh. ".

Tabela 2 - Relação entre circunferência abdominal e risco de morbidades associadas à obesidade em mulheres

\begin{tabular}{cc}
\hline $\begin{array}{c}\text { CA } \\
(\mathbf{c m})\end{array}$ & Risco de complicações metabólicas (mulheres) \\
$\mathbf{2 8 0}$ & Aumentado \\
\hline$\geq \mathbf{8 8}$ & Aumentado substancialmente \\
\hline \multicolumn{2}{c}{ Fonte: Organização Mundial de Saúde (2000). }
\end{tabular}

Tabela 3 - Relação entre circunferência abdominal e risco de morbidades associadas à obesidade em homens

\begin{tabular}{cc}
\hline $\begin{array}{c}\text { CA } \\
(\mathbf{c m})\end{array}$ & $\begin{array}{c}\text { Risco de complicações metabólicas } \\
\text { (homens) }\end{array}$ \\
\hline $\mathbf{8 0}$ & Aumentado \\
$\geq \mathbf{8 8}$ & Aumentado substancialmente
\end{tabular}

Fonte: dados da Organização Mundial de Saúde (OMS) eөlocades em Micreseft Excel.

Em virtude do apresentado, são visíveis os benefícios para a triagem de pacientes por meio do uso da circunferência abdominal e do IMC, um dos indicadores usados pela Organização Mundial de Saúde (OMS) para avaliação do estado nutricional.

\section{MATERIAIS E MÉTODOS}

A análise retrospectiva de prontuários foi a metodologia utilizada como fonte de pesquisa de dados sobre cada paciente, e a elaboração de um banco de dados com as informações dos pacientes construído por meio de projeto de pesquisa aprovado no CEP da instituição 1.620.200. Esse banco de dados, foi necessário para repassar as relações familiares entre os pacientes ali presentes para a construção dos heredogramas com uma segunda ferramenta.

Adicionalmente, ação de extensão foi realizada em 2018.1 no setor de Endocrionologia do Hospital Universitário Walter Cantídio (HUWC) o qual disponibilizou auxiliares administrativos, e contou com o auxílio de cirurgiã de Cabeça e Pescoço do HUWC. O mutirão visou analisar a necessidade atual de paratireoidectomia desses pacientes, visto que muitos esperam há anos pela operação e as necessidades de alguns se agravaram com o passar do tempo. Uma lista para chamada dos pacientes com necessidade de paratireoidectomia foi elaborada pelo setor de Endocrinologia e o HUWC fez o contato com os citados. 
$\mathrm{Na}$ ação, foram atendidos 17 pacientes, dos quais 10 eram mulheres e 7, homens. Primeiramente, aplicou-se questionário para coleta de novos dados - como litíase renal, osteoporose, cálcio iônico —, e foi abordado, entre outros aspectos, a formação familiar do paciente e a existência de casos na família com algum dos sintomas típicos da NEM-1. Em seguida, o grupo foi avaliado quanto ao peso, à altura e à circunferências abdominal, seguindo para avaliação por médica cirurgiã de Cabeça e Pescoço. Por fim, estudando a situação atual de cada paciente, foi feita uma lista para paratireoidectomia em ordem de prioridade, repassada ao setor de Cabeça e Pescoço. Participaram da ação: a cirurgiã do setor de Cabeça e Pescoço do HUWC, sete membros do NEPTUNEF e sua orientadora, Dra. Ana Rosa Pinto Quidute.

A confecção dos heredogramas foi por meio da utilização do software GenoPro®. O programa dispõe de várias opções e métodos de edição de para a construção de heredogramas, o que facilita a observação da distribuição patológica da neoplasia entre os pacientes portadores.

Através desses dois programas computacionais, foi realizado a elaboração de um banco de dados para o armazenamento de diversos dados referentes ao diagnóstico da patologia, tais como os dados metabólicos e antropométricos, a recorrência cirúrgica dos diferentes tumores, densitometria óssea, sintomas iniciais e exames de imagem, como forma de otimizar as informações obtidas pelo prontuário, facilitando tanto o trabalho de pesquisa, quanto a abordagem do estudo médico em cada família.

Terminado o banco de dados, foi feito a confecção de 14 heredogramas, sendo 8 baseados nas primeiras famílias diagnosticadas no SED do HUWC, enquanto que os outros 6 foram atualizações dessas famílias com a adição de novos membros cujo diagnóstico de NEM1 foi fechado nos últimos 10 anos. A construção desses heredogramas baseados em um intervalo de 10 anos permitiu a verificação do aumento do número de casos diagnosticados e o a redução da idade ao diagnóstico desses pacientes através do screening familiar e a investigação de casos suspeitos em famílias com algum membro já acometido.

\section{RESULTADOS E DISCUSSÕES}

Em meio às probabilidades de herança em doenças genéticas, a equipe de saúde precisa de ferramentas para um estudo mais aperfeiçoado e especializado para cada caso. A criação de heredogramas está possibilitando, atualmente, um maior número de famílias com seus casos mapeados possibilitando também que as equipes de pesquisa consigam uma interpretação mais 
prática da distribuição das comorbidades causadas pela doença dentro de cada grupo familiar.

O SED do HUWC iniciou seus serviços ambulatoriais especializados em NEM-1 no ano de 2008 e, nesse ano, já haviam sido diagnosticados 32 pacientes distribuídos em 8 famílias. Atualmente, o número de casos foi elevado para um total de 98 pacientes distribuídos em 13 famílias e 2 casos isolados, em 2018. O aumento do número de pacientes é consequência direta da utilização de heredogramas para a avaliação familiar, pois, através do diagnóstico clínico, que consiste na presença de duas ou mais comorbidades típicas da NEM-1, de um paciente, sua família pode então ser solicitada para a realização de exames que possam diagnosticar a doença que, por apresentar um padrão de herança autossômico dominante, se manifesta em $50 \%$ dos casos.

Dentre os resultados possibilitados pela utilização dos heredogramas, três famílias se destacaram por apresentações peculiares. Tanto o diagnóstico de pacientes com uma idade cada vez mais cedo como a predição de ligação direta entre as causas de óbitos que, até então, não tinha nenhuma relação com a doença foram possíveis interpretações.

Na primeira família, foram analisados retrospectivamente os dados de 24 indivíduos. 18 foram diagnosticados clinicamente como portadores de NEM-1 e 8, até a última avaliação, não apresentavam história clínica diagnosticada. Dos diagnosticados, houve um total de 4 óbitos, dos quais 1 apresenta relação direta com a doença (metástase hepática de TGEP), e aos outros 3 casos não foi possível associar o óbito com qualquer manifestação da NEM-1. Dos 18 casos, 11 apresentaram HPT, 10 AH e 8 TGEP. A média de idade foi de 46,5 $\pm 12,4$ anos (21 a 69 anos). Casos de surdo mudez congênita foram identificados.

$\mathrm{Na}$ segunda família, ocorreu um fato interessante através da utilização dos heredogramas, pois, por meio de uma paciente que, aparentemente não possuía qualquer relação com outra família, foi comprovado que sua família era, na verdade, um subgrupo de parentes dentro de uma família inicial. Através dessa situação foi possível ainda fazer uma integração entre os centros de atendimento em endocrinologia da região, pois, a última família que foi integrada era acompanhada no Hospital Geral de Fortaleza (HGF). Em relação às comorbidades da NEM-1, a família apresentou um total de 5 pacientes com diagnóstico comprovado, sendo distribuído entre 3 casos de HPT, 3 TGEP e 1 AH. A média de idade foi acima do relatado na literatura, com resultado de $52 \pm 9$ anos (39 a 64 anos).

A última família que apresentou características importantes através do estudo por meio de heredogramas foi a terceira, na qual 10 pacientes tiveram diagnóstico conclusivo para NEM- 
1. A distribuição de casos se deu em 3 pacientes com HPT, 8 AH e 4 TGEP. A média de idade foi de 47,8 $\pm 11,7$ (33 a 67 anos).

Já na ação extensionista de mutirão realizada pelo NEPTUNEF no HUWC, constatouse um antecessor comum entre duas famílias aparentemente não relacionadas, uma das quais supunha-se ter origem fora do estado do Ceará. Desse modo, demonstrou-se a origem comum dessas famílias em região já estudada pelo NEPTUNEF. Detectamos também a presença de síndrome de surdo mudez em um certo número dos pacientes atendidos. No mutirão, avaliouse 17 pacientes, dos quais $35 \%$ obesos, $59 \%$ com sobrepeso e $41 \%$ com humor deprimido. Dos atendidos, 10 eram mulheres de idade 45,1 $\pm 2,8$ anos (16 a 59 anos), altura 1,53 $\pm 0,05 \mathrm{~m}(1,47$ a 1,69m), peso $62,66 \pm 3,8 \mathrm{~kg}(47$ a $87,8 \mathrm{~kg}), \operatorname{IMC} 26,8 \pm 0,2 \mathrm{~kg} / \mathrm{m}^{2}\left(21,46\right.$ a $\left.34,3 \mathrm{~kg} / \mathrm{m}^{2}\right)$ e circunferência abdominal 88,6 $\pm 2,8 \mathrm{~cm}$ (72 a $106 \mathrm{~cm})$. Os homens acompanhados na atividade somaram 7, tendo idade 49,9 $\pm 27,6$ anos (23 a 72 anos), altura 1,62 $\pm 0,2 \mathrm{~m}(1,48$ a 1,77m), peso $76,3 \pm 65,3 \mathrm{~kg}(39,6$ a $131,8 \mathrm{~kg})$ e IMC $28,5 \pm 17 \mathrm{~kg} / \mathrm{m}^{2}\left(18 \mathrm{a} 42 \mathrm{~kg} / \mathrm{m}^{2}\right)$. Dos 7 homens, 6 tiveram a circunferência abdominal aferida, sendo ela de 96,8 $\pm 18,4 \mathrm{~cm}(74 \mathrm{a} 116 \mathrm{~cm})$.

Tabela 4 - Valores antropométricos das mulheres atendidas no mutirão do NEPTUNEF

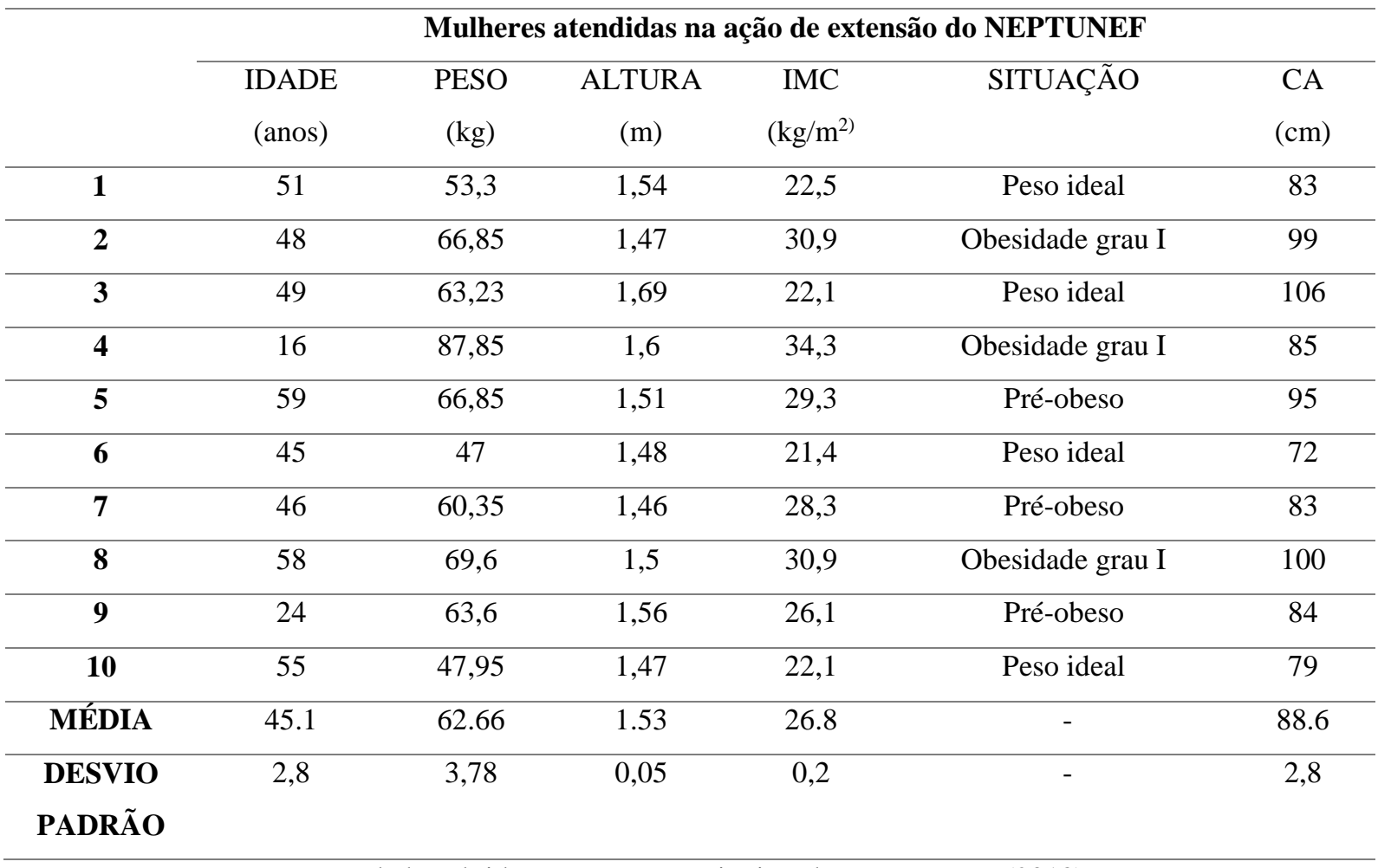

Fonte: dados obtidos na ação extensionista do NEPTUNEF (2018). 
Tabela 5 - Valores antropométricos dos homens atendidos no mutirão do NEPTUNEF

\begin{tabular}{|c|c|c|c|c|c|c|}
\hline & \multicolumn{6}{|c|}{ Homens atendidos na ação de extensão do NEPTUNEF } \\
\hline & $\begin{array}{c}\text { IDADE } \\
\text { (anos) }\end{array}$ & $\begin{array}{c}\text { PESO } \\
(\mathrm{kg})\end{array}$ & $\begin{array}{c}\text { ALTURA } \\
\text { (m) }\end{array}$ & $\begin{array}{c}\text { IMC } \\
\left(\mathrm{kg} / \mathrm{m}^{2}\right)\end{array}$ & SITUAÇÃO & $\begin{array}{l}\mathrm{CA} \\
(\mathrm{cm})\end{array}$ \\
\hline 1 & 71 & 39,45 & 1,48 & 18 & Baixo peso & 74 \\
\hline 2 & 36 & 89,4 & 1,62 & 34 & Obesidade grau I & 115 \\
\hline 3 & 72 & 75,85 & 1,58 & 30,4 & Obesidade grau I & 116 \\
\hline 4 & 64 & 59,2 & 1,57 & 24 & Peso ideal & 92 \\
\hline 5 & 23 & 68,75 & 1,675 & 24,5 & Peso ideal & 84 \\
\hline 6 & 51 & 69,9 & 1,63 & 26,3 & Pré-obeso & 100 \\
\hline 7 & 32 & 131,85 & 1,77 & 42,1 & Obesidade grau III & \\
\hline MÉDIA & 49,9 & 76,3 & 1,62 & 28.5 & - & 96.8 \\
\hline $\begin{array}{l}\text { DESVIO } \\
\text { PADRÃO }\end{array}$ & 27,6 & 65,3 & 0,2 & 17 & - & 18,4 \\
\hline
\end{tabular}

Fonte: dados obtidos na ação extensionista do NEPTUNEF (2018).

\section{CONCLUSÃO}

Verificamos a importância da construção do heredograma para o estudo de uma doença hereditária onde a análise dos dados clínicos ajudou a caracterizar os portadores da síndrome, facilitando, portanto, a abordagem da equipe multidisciplinar de saúde no diagnóstico e acompanhamento dos pacientes e famílias com acometimento devido a doença hereditária.

A experiência mostrada no SED do HUWC demonstra a necessidade de uma maior capacitação com recursos digitais e informáticos na prática clínica dos médicos. A confecção dessas árvores genealógicas através de programas como o GenoPro® podem diminuir o tempo de elaboração desses heredogramas, otimizar a compreensão dos pacientes acometidos dentro de uma família e prever os pacientes que precisam ser investigados dentro de um padrão clínico.

Em meio a rotina corrida, muitos profissionais da área podem deixar passar o fato que essas doenças podem acometer vários pacientes da mesma família, o que pode dificultar o quadro de identificação de um futuro paciente ou mesmo aumentar o tempo entre o início de sintomas e o diagnóstico.

Programas computacionais de baixo custo, como o GenoPro®, são fundamentais no cotidiano atual de médicos em qualquer especialidade que possa se deparar com síndromes genéticas. No caso do serviço de endocrinologia, a utilização da ferramenta foi eficaz tanto no mapeamento dessa doença, como na identificação de outras patologias associadas, sendo um 
exemplo da necessidade desse tipo de implementação.

Em virtude da alta prevalência de obesidade e sobrepeso na amostra atendida no mutirão realizado pelo NEPTUNEF no HUWC detectada por meio de medidas antropométricas, tais como, peso, altura — permitindo o cálculo do IMC — e circunferência abdominal, é visível a necessidade de um olhar crítico e atento dos profissionais da saúde que participam do acompanhamento de pacientes, pois, como foi falado anteriormente, esse excesso de gordura corporal aumenta o risco de câncer, o que é agravado pela tendência genética a certas neoplasias nos portadores de NEM-1. Com base na zona de risco indicada pelo IMC, é possível, com orientações básicas desses profissionais, orientar o paciente para uma mudança de hábitos — incluindo exercícios compatíveis com a faixa etária e as condições físicas do indivíduo, além de uma dieta adequada às condições socioeconômicas e cotidianas de cada ser humano - ou, em casos mais críticos de obesidade, pesquisar a necessidade ou não de tratamento medicamentoso ou cirúrgico para a perda de peso.

A detecção precoce do sobrepeso, por meio da triagem com medidas de fácil acesso, rápidas e de despesa mínima, como as citadas acima, visam, sobretudo, a prevenção do desenvolvimento de outras comorbidades, do agravamento das já existentes, e a redução do risco de neoplasias, que merecem uma atenção especial, principalmente, em portadores dessa síndrome neoplásica, a NEM-1.

\section{REFERÊNCIAS}

ANJOS, Luiz A. et al. Índice de massa corporal (massa corporal.estatura-2) como indicador do estado nutricional de adultos: revisão da literatura. Revista de Saúde Pública, [s.1.], v. 26, n. 6, p.431-436, dez. 1992. FapUNIFESP (SciELO). http://dx.doi.org/10.1590/s003489101992000600009 .

CALLE, Eugenia E.; KAAKS, Rudolf. Overweight, obesity and cancer: epidemiological evidence and proposed mechanisms. Nature Reviews Cancer, [s.1.], v. 4, n. 8, p.579-591, ago. 2004. Springer Nature. http://dx.doi.org/10.1038/nrc1408.

CUNHA, Hercio Azevedo de Vasconcelos et al. Associação da obesidade à presença de comorbidades decorrentes em trabalhadores do Hospital e Maternidade Celso Pierro. Revista de Ciências Médicas, Campinas, v. 19, n. 1-6, p.23-31, 2010.

GOUDET, P. et al. Risk factors and causes of death in MEN1 disease. A GTE (Groupe d'Etude des Tumeurs Endocrines) cohort study among 758 patients. World J Surg, v. 34, n. 2, p. 249-55, Feb 2010.

IMAMURA, M. Recent standardization of treatment strategy for pancreatic neuroendocrine 
tumors. World J Gastroenterol, v. 16, n. 36, p. 4519-25, Sep 28, 2010.

ISHIDA, E. et al. Attenuated expression of menin and p27(kip1) in an aggressive case of multiple endocrine neoplasia type 1 (MEN1) associated with an atypical prolactinoma and a malignant pancreatic endocrine tumor. Endocrin. J., 58, 287- 296, 2011.

LIPS, C. J.; DREIJERINK, K. M.; HOPPENER, J. W. Variable clinical expression in patients with a germline MEN1 disease gene mutation: clues to a genotype- phenotype correlation. Clinics (Sao Paulo), v. 67 Suppl 1, p. 49-56, 2012.

LOURENCO, D. M., JR. et al. Early-onset, progressive, frequent, extensive, and severe bone mineral and renal complications in multiple endocrine neoplasia type 1- associated primary hyperparathyroidism. J Bone Miner Res, v. 25, n. 11, p. 2382- 91, Nov 2010.

LOURENCO, D. M., JR. et al. Multiple endocrine neoplasia type 1 in Brazil: MEN1 founding mutation, clinical features, and bone mineral density profile. Eur J Endocrinol, v. 159 , n. 3, p. 259-74, Sep 2008.

MARX, S. J. Molecular genetics of multiple endocrine neoplasia types 1 and 2. Nat Rev Cancer, v. 5, n. 5, p. 367-375, 05//print 2005.

NORTON, J. A.; JENSEN, R. T. Current surgical management of Zollinger-Ellison syndrome (ZES) in patients without multiple endocrine neoplasia-type 1 (MEN1). Surg Oncol, v. 12, n. 2, p. 145-51, Aug 2003.

PEIXOTO, Maria do Rosário Gondim et al . Circunferência da cintura e índice de massa corporal como preditores da hipertensão arterial. Arq. Bras. Cardiol., São Paulo, v. 87, n. 4, p. 462-470, Oct. 2006. Disponível em:

$<$ http://www.scielo.br/scielo.php?script=sci_arttext\&pid=S0066-

782X2006001700011\&lng=en\&nrm=iso>. Acesso em: 31 ago. 2018.

SOUZA, M.R. et al. Multiple endocrine neoplasia type 1: what has changed after 6 years screening new cases. Archives of Endocrinology and Metabolism, v.59, supp.1, p. 82, May 2015.

THAKKER, R. V. Multiple endocrine neoplasia type 1 (MEN1) and type 4 (MEN4). Mol Cell Endocrinol, v. 386, n. 1-2, p. 2-15, Apr 52014.

THAKKER, R. V. Multiple endocrine neoplasia type 1. Indian J Endocrinol Metab, v. 16, n. Suppl 2, p. S272-4, Dec 2012.

THAKKER, R. V. et al. Clinical practice guidelines for multiple endocrine neoplasia type 1 (MEN1). J Clin Endocrinol Metab, v. 97, n. 9, p. 2990-3011, Sep 2012.

TONELLI, F. et al. Surgical approach in patients with hyperparathyroidism in multiple endocrine neoplasia type 1: total versus partial parathyroidectomy. Clinics (Sao Paulo), v. 67 Suppl 1, p. 155-60, 2012. 
TONELLI, F. et al. Gastroenteropancreatic neuroendocrine tumors in multiple endocrine neoplasia type 1. Cancers (Basel), v. 4, n. 2, p. 504-22, 2012.

World Health Organization. Body mass index classification - report of a WHO consultation on obesity. Geneva: WHO, 1995. Technical Report Series 854.

World Health Organization. Obesity: preventing and managing the global epidemic. Report of a World Health Organization Consultation. Geneva: World Health Organization, 2000. p. 256.

WHO. Technical Report Series 284.

http://portalms.saude.gov.br/component/content/article/804-imc/40508-so-o-imc-nao-dizcomo-voce-esta. Acesso em: 01 set. 2018. 